\section{Utilização dos serviços ambulatoriais de saúde por mulheres: estudo de base populacional no Sul do Brasil}

\author{
Use of outpatient health services by women: \\ a population-based study in southern Brazil
}

\begin{abstract}
The aim of this study was to verify the use of outpatient health services and associated factors among women ages 20 to 60 years living in the urban area of São Leopoldo, Rio Grande do Sul State, Brazil. This was a cross-sectional, population-based study with a sample of 1,026 women in 2003. The association between the independent variables and the outcome was evaluated by means of prevalence ratios and 95\% confidence intervals (95\%CI). Poisson regression was used with a hierarchical model. Among 1,022 women, 136 (13.3\%; 95\% CI: 11.2-15.4) had not used outpatient services in the 12 months prior to the interview. Women in lower socioeconomic classes $(C, D$, and $E)$, with less schooling, and with lower income showed lower use of outpatient health services. Thus, the most vulnerable women and those with the greatest health needs showed the lowest use of health services, suggesting unfavorable conditions in the local health system and pointing to the presence of lack of universal care and equal access
\end{abstract}

Ambulatory Health Services; Health Services Accessibility; Women
Juvenal Soares Dias-da-Costa 1,2

Adriana Dewes Presser 2

Anelise Fernanda Zanolla ${ }^{2}$

Daiane Giacomet Ferreira 2

Gabriela Perozzo ${ }^{2}$

Ivani Bueno de Almeida Freitas 2

Livia Tavares Portolan 2

Renata Tôndolo Tavares 2

Maria Teresa Anselmo Olinto 2

Marcos Pascoal Pattussi 2

\section{Introdução}

Estudos têm mostrado o crescimento do número dos estabelecimentos de saúde públicos no Brasil a partir do processo de Reforma Sanitária. Dados da pesquisa sobre assistência médico-sanitária demonstraram que o número de serviços sem internação no Brasil, de 5.805 estabelecimentos, em 1976, passou para 41.200 em $2005^{1}$. O crescimento da rede pública no Brasil desde a implantação do processo de Reforma Sanitária também foi destacado por Pestana \& Mendes 2 (p. 24) “no período 1992/1999, o número de estabelecimentos públicos cresceu 24,9\%, enquanto que os estabelecimentos privados tiveram uma redução de $2,8 \%$. Dentre os serviços públicos em geral - com e sem internação -, destaca-se a participação dos estabelecimentos municipais que passaram de 69\% em 1992 para 92\% em 1999". Esse incremento expressou o direito fundamental do ser humano estabelecido na Constituição Federal, no artigo 196, e na Lei $n^{\circ} .8 .080$ (Lei Orgânica da Saúde, de 19 de setembro de 1990) em seu parágrafo primeiro quando foram enunciadas as políticas que visavam ao acesso universal e igualitário aos serviços de saúde.

O conceito de acesso aos serviços de saúde de acordo com Travassos \& Martins 3 é abrangente, a terminologia utilizada por alguns autores como uso dos serviços, desempenho do sistema, resultado dos cuidados tem sido tão complexa e ampla quanto o conceito de saúde, não se expli- 
cando unicamente pelo uso de serviços de saúde. Esses autores têm recomendado a verificação do efeito de múltiplos fatores, seu contexto e as variações no uso de serviços, bem como as etapas no processo de utilização dos serviços como forma de orientação na formulação de políticas ${ }^{3}$.

Estudos epidemiológicos têm mostrado alguns fatores que interferem no uso dos serviços de saúde como: variações geográficas, sociais, necessidades individuais, qualidade de vida, nível de conhecimento sobre saúde, associando-se fatores como sexo, idade, morbidade e condições econômicas 4,5,6,7,8. Além disso, estudo sobre a descrição dos fatores de risco e do impacto social das doenças crônicas não transmissíveis no Brasil mostrou a concentração de doenças nas regiões Sul e Sudeste, o envelhecimento da população e o aumento da prevalência de incapacidades, invalidez e perda da autonomia 9 , fatores que ampliam a dimensão do problema e a necessidade de reorganização dos sistemas e serviços de saúde. No contexto de caracterizar o uso de serviços de saúde, outros estudos realizados no Brasil apontaram para a concentração de procedimentos por habitante/ano e cobertura assistencial nas regiões Sul e Sudeste 10; assim como evidenciaram modificações nos sistemas de saúde determinados por diferenças sócio-econômicas e graus de utilização dos serviços 11. Assim, estudos epidemiológicos sobre a utilização de serviços de saúde podem auxiliar o planejamento em saúde no sentido de propiciar o acesso universal e igualitário dos serviços e na reorganização dos sistemas.

O presente artigo teve como objetivo verificar a utilização pelas mulheres dos serviços ambulatoriais de saúde no Município de São Leopoldo, Rio Grande do Sul, Brasil, e fatores associados.

\section{Materiais e métodos}

Este estudo transversal faz parte do projeto de pesquisa Condições de Saúde das Mulheres: Estudo de Base Populacional na Região do Vale do Rio dos Sinos, realizado pela Universidade do Vale do Rio dos Sinos (UNISINOS) através do Programa de Pós-graduação, Mestrado em Saúde Coletiva, no ano de 2003. Neste trabalho, buscou-se caracterizar a saúde das mulheres residentes na zona urbana de São Leopoldo, na faixa etária de 20 a 60 anos. O projeto de pesquisa foi submetido e aprovado pelo Comitê de Ética e Pesquisa da Faculdade de Medicina da Universidade Federal de Pelotas.

A cidade de São Leopoldo situa-se na Região Metropolitana de Porto Alegre, no Vale do Rio dos Sinos, no Estado do Rio Grande do Sul. O muni- cípio tinha, segundo o último censo demográfico (Instituto Brasileiro de Geografia e Estatística. Censo Demográfico 2000; http://www.ibge.gov. br), 193.547 habitantes, sendo 98.781 mulheres (51,07\%).

Foi realizada uma amostra por conglomerados, sendo sorteados quarenta setores censitários entre os 270 existentes na zona urbana da cidade de São Leopoldo. Cada um desses setores teve um quarteirão sorteado. $\mathrm{O}$ quarteirão teve suas esquinas identificadas, e, assim, o ponto de partida foi igualmente sorteado, garantindo-se aleatoriedade na coleta de dados. Ao se entrevistar as pessoas da faixa etária em determinado domicílio, as próximas duas casas eram saltadas. Dessa forma, para que a totalidade da amostra fosse atingida, foram visitados 36 domicílios em cada setor sorteado. A amostra foi estimada a partir de diferentes desfechos previstos neste projeto, escolhendo-se aquele com maior número de indivíduos (prevalência de diabetes mellitus). Calculou-se um tamanho de amostra que permitisse identificar uma razão de risco de 2,0, para um nível de confiança de $95 \%$, de um poder de $80 \%$, mantida a razão de não expostos:expostos de 1:3. Entre os diferentes desfechos incluídos no projeto, optou-se pelo que exigia maior tamanho: 1.086 pessoas. Foram adicionados ao tamanho da amostra mais $10 \%$ de possíveis perdas e recusas e $15 \%$ para controlar fatores de confusão na análise dos dados, totalizando 1.358 pessoas ${ }^{12}$. Conhecendo-se o número de pessoas necessário para a amostra, a média de pessoas por domicílio na cidade de São Leopoldo $(3,56)$, a proporção de mulheres na faixa etária de interesse (30\%), foram visitadas 1.440 residências.

Foram utilizados questionários padronizados, pré-codificados e pré-testados. O grupo de entrevistadoras foi composto por acadêmicas de cursos na área da saúde da UNISINOS. Os entrevistadores foram treinados para procura de mulheres não encontradas (três visitas ao mesmo domicílio em horários diferentes), para a aplicação do instrumento e coleta das medidas de peso, altura e pressão arterial. Também foi realizado teste piloto para treinamento das entrevistadoras, e os dados foram coletados entre março e dezembro de 2003.

O controle de qualidade foi realizado em uma amostra aleatória de $10 \%$ das pessoas incluídas no estudo.

Após a finalização da coleta dos dados, foram encontradas 1.086 mulheres de 20 a 60 anos; dessas, $58(5,3 \%)$ foram classificadas como perdas e recusas, sendo incluídas no estudo 1.026 pessoas entrevistadas.

O uso de serviços de saúde na cidade de São Leopoldo foi medido a partir de resposta afirma- 
tiva sobre a utilização de algum tipo de serviço ambulatorial de saúde nos 12 meses que antecederam a entrevista.

Foram analisadas como variáveis sócio-econômicas: classe econômica segundo a Associação Nacional de Empresas de Pesquisa (ANEP) 13, renda familiar per capita e escolaridade das mulheres. A classe econômica da ANEP está fundamentada a partir da posse de determinados bens materiais, escolaridade do chefe da família e número de empregados na família, a partir da construção de um escore com pontos de corte bem definidos para cada categoria 13 .

As características demográficas incluídas no estudo foram: cor da pele observada, idade e estado civil.

Entre os hábitos de vida, foram analisadas as seguintes variáveis: tabagismo, atividade física e uso de álcool. Foram classificadas como sedentárias as mulheres que praticavam atividade física uma vez por semana ou tinham atividades diárias consideradas de baixo consumo energético; e não sedentárias ou ativas as que praticavam duas ou mais vezes por semana, no mínimo dez minutos de duração 14 . O consumo de álcool foi estabelecido a partir da freqüência, tipo de bebida e quantidade ingerida. Sendo que, o consumo abusivo de álcool foi definido a partir da ingestão de $30 \mathrm{~g} /$ dia de etanol ou mais 15 .

Entre as morbidades, foram analisadas as variáveis: presença de diabetes mellitus, hipertensão arterial, excesso de peso e distúrbios psiquiátricos menores. A presença de diabetes mellitus foi definida a partir de diagnóstico médico referido pelas mulheres. A pressão arterial de cada mulher incluída no estudo foi medida duas vezes durante a aplicação do questionário. As medidas das pressões sistólicas e diastólicas corresponderam às suas médias aritméticas e foram efetuadas no meio e no final do questionário. Foram utilizados estetoscópio e esfigmomanômetro aneróide (BD - Brasil, Curitiba, Brasil) para se efetuar a medida no braço direito, estando a entrevistada sentada, sem fumar, consumir café, chimarrão ou outro alimento qualquer durante a entrevista. Hipertensão arterial sistêmica foi classificada de acordo com os níveis tensionais da Joint National Committee (JNC) e/ou com o uso de medicação anti-hipertensiva. Portanto, as mulheres com níveis tensionais iguais ou maior de $140 \mathrm{x}$ $90 \mathrm{mmHg}$ e/ou com medidas tensionais abaixo desses níveis, mas com uso de medicação antihipertensiva foram classificadas como hipertensas 16. A coleta das medidas antropométricas foi realizada por meio de balança Sunrise com precisão de 100g (Proplastik, São Paulo, Brasil) e altura (cm) com antropômetro com precisão de $0,1 \mathrm{~cm}$ (confeccionado na marcenaria da UNISINOS).
As duas medidas foram coletadas duas vezes. Verificou-se o estado nutricional pelo cálculo do índice de massa corporal $\left[\mathrm{IMC}=\right.$ peso $(\mathrm{kg}) /$ altura $^{2}$ (m)], sendo consideradas com excesso de peso as mulheres com IMC $\geq 25,0 \mathrm{~kg} / \mathrm{m}^{2}$. A presença de distúrbios psiquiátricos menores foi avaliada por meio do índice do Self Reporting Questionaire-20 Items (SRQ-20), que foi concebido para detectar psicopatologias psiquiátricas em atenção primária à saúde com fins de investigação e não de diagnóstico. $\mathrm{O}$ ponto de corte estabelecido para caracterizar a presença de distúrbios psiquiátricos menores foi 717 .

A entrada dos dados foi realizada através do programa Epi Info (Centers for Disease Control and Prevention, Atlanta, Estados Unidos), em dupla entrada com posterior comparação, para se eliminar a probabilidade de erros de digitação. A análise univariada e bivariada dos dados foi realizada pelo programa SPSS versão 11.0 (SPSS Inc., Chicago, Estados Unidos). O efeito de delineamento e a correção das medidas de efeito, com respectivos intervalos de confiança e testes de associação, foram calculados no programa Stata versão 7.0 (Stata Corp., College Station, Estados Unidos). A análise multivariável foi realizada pela regressão de Poisson no programa Stata. Optouse pela regressão de Poisson pela possibilidade de demonstrar a magnitude das associações sem problemas de estimativa, oriundas da utilização de odds ratio $(\mathrm{OR}){ }^{18}$. As variáveis foram dispostas em modelo hierarquizado ${ }^{19}$, o que significa que as variáveis mais distais determinam o grupo de variáveis intermediárias e o desfecho (Figura 1). Assim, as variáveis que ingressaram no modelo atingiram valor de $\mathrm{p}<0,20$ na análise bivariada. Entretanto, apenas se mantiveram no modelo final quando a significância estatística foi valor de $\mathrm{p}<0,05$.

As variáveis sócio-econômicas incluídas nesse estudo tinham relação entre si. Por exemplo, escolaridade está incluída na classificação de classe econômica da ANEP e, a partir da renda, determina o acúmulo de bens materiais. Assim, na análise multivariável, foram utilizadas as três variáveis sócio-econômicas como modelos diferentes.

\section{Resultados}

Entre as 1.022 mulheres incluídas no estudo, 136 (13,3\%; IC95\%: 11,2-15,4) não utilizaram os serviços ambulatoriais de saúde em São Leopoldo durante o ano de 2003.

Quanto aos fatores sócio-econômicos, a maioria das mulheres tinham renda mensal per capita de até três salários mínimos (78,9\%) e, pre- 


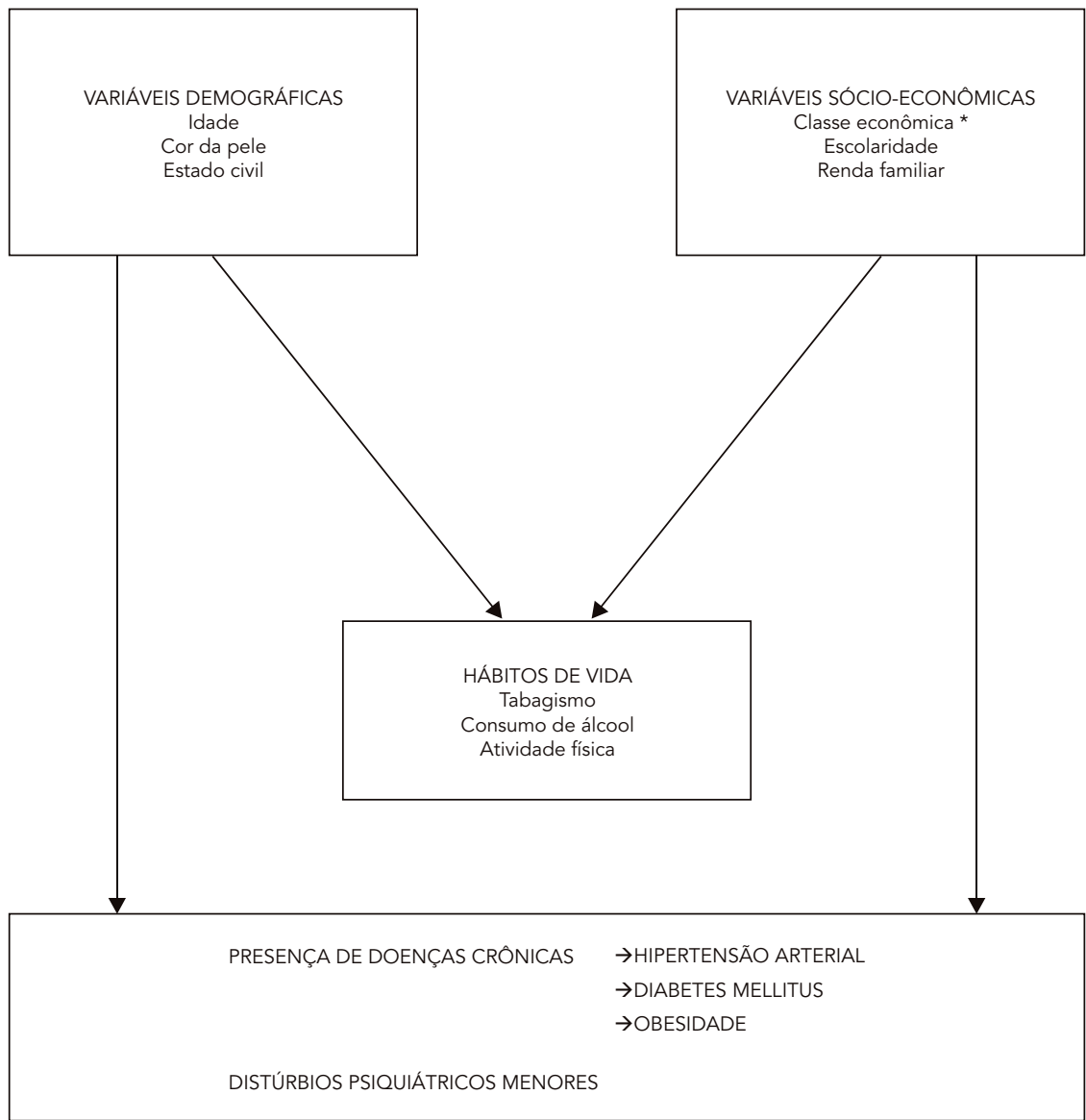

USO DE SERVIÇOS DE SAÚDE

* Segundo a Associação Nacional de Empresas de Pesquisa (ANEP) 13.

dominantemente, pertenciam à classe econômica C (39,3\%). Em relação à escolaridade, 44,5\% apresentavam menos de sete anos de estudo (Tabela 1). Observou-se que cerca de metade da amostra estava na faixa etária de 20 a 39 anos, constatando-se predomínio de mulheres de cor da pele branca (84\%) e das casadas ou vivendo em união $(64,1 \%)$ (Tabela 2). Quanto aos hábitos de vida, a maioria das mulheres era sedentária $(68,5 \%)$, nunca havia fumado (59\%) e referia ingestão de álcool inferior a 30g por dia $(94,1 \%)$. Entre as morbidades investigadas, constatou-se que $12,7 \%$ das mulheres apresentavam hiperten- são, e 3,6\%, diabetes. Aproximadamente, metade da amostra estudada tinha excesso de peso, e $42,6 \%$ das mulheres apresentavam distúrbios psiquiátricos menores (Tabela 3).

A análise mostrou teste de tendência linear significativa em todas as variáveis sócio-econômicas. Assim, à medida que diminuíam os níveis de classe econômica, de escolaridade e de renda familiar, aumentava o percentual de mulheres que não consultavam. No entanto, as medidas de estimativa foram diferentes apenas em relação às classes econômicas D e E (IC95\%: 1,01-5,92) e à faixa de renda menor que um salário mínimo 
Algumas características das mulheres que não utilizam serviços de saúde segundo variáveis sócio-econômicas. São Leopoldo, Rio Grande do Sul, Brasil, 2003.

\begin{tabular}{|c|c|c|c|c|c|}
\hline Variável & n (\%) & $\begin{array}{c}\text { Prevalência de } \\
\text { não uso (\%) }\end{array}$ & $\begin{array}{c}\text { Razão de } \\
\text { prevalência }\end{array}$ & IC95\% & Valor de $p$ \\
\hline Classe econômica * & & & & & 0,001 \\
\hline A & $73(7,2)$ & $5(6,8)$ & 1,00 & - & \\
\hline B & $265(26,0)$ & $21(7,9)$ & 1,16 & $0,45-2,96$ & \\
\hline C & $400(39,3)$ & $63(15,8)$ & 2,30 & $0,96-5,52$ & \\
\hline$D+E$ & $281(27,6)$ & $47(16,7)$ & 2,44 & $1,01-5,92$ & \\
\hline Escolaridade (anos) & & & & & $<0,001$ \\
\hline 12 ou mais & $401(39,2)$ & $33(8,2)$ & 1,00 & - & \\
\hline $8-11$ & $167(16,3)$ & $26(15,6)$ & 1,89 & $1,17-3,06$ & \\
\hline $5-7$ & $247(24,2)$ & $40(16,2)$ & 1,97 & $1,28-3,03$ & \\
\hline $0-4$ & $207(20,3)$ & $37(17,9)$ & 2,17 & $1,40-3,37$ & \\
\hline Renda familiar (salários mínimos) & & & & & $<0,001$ \\
\hline 6,01 ou mais & $73(7,2)$ & $5(6,8)$ & 1,00 & - & \\
\hline $3,01-6$ & $140(13,9)$ & $8(5,7)$ & 0,83 & $0,28-2,46$ & \\
\hline $1,01-3$ & $426(42,2)$ & $56(13,1)$ & 1,92 & $0,80-4,63$ & \\
\hline $0-1$ & $371(36,7)$ & $66(17,8)$ & 2,60 & $1,08-6,22$ & \\
\hline
\end{tabular}

* Segundo a Associação Nacional de Empresas de Pesquisa (ANEP) 13.

Tabela 2

Algumas características das mulheres que não utilizam serviços de saúde segundo variáveis demográficas. São Leopoldo, Rio Grande do Sul, Brasil, 2003.

\begin{tabular}{|c|c|c|c|c|c|}
\hline Variável & n (\%) & $\begin{array}{l}\text { Prevalência de } \\
\text { não uso (\%) }\end{array}$ & $\begin{array}{c}\text { Razão de } \\
\text { prevalência }\end{array}$ & IC95\% & Valor de $p$ \\
\hline Idade (anos) & & & & & 0,91 \\
\hline $20-29$ & $282(27,6)$ & $33(11,7)$ & 1,00 & - & \\
\hline $30-39$ & $254(24,9)$ & $36(14,2)$ & 1,21 & $0,78-1,88$ & \\
\hline $40-49$ & $301(29,5)$ & $48(15,9)$ & 1,36 & $0,90-2,06$ & \\
\hline $50-60$ & $185(18,1)$ & $19(10,3)$ & 0,88 & $0,51-1,50$ & \\
\hline Cor da pele & & & & & 1,0 \\
\hline Branca & $858(84,0)$ & $114(13,3)$ & 1,00 & - & \\
\hline Não branca & $164(16,0)$ & $22(13,4)$ & 1,01 & $0,66-1,54$ & \\
\hline Estado civil & & & & & 0,37 \\
\hline Casada e em união & $655(64,1)$ & $82(12,5)$ & 1,00 & - & \\
\hline Viúvas, separadas e solteiras & $367(35,9)$ & $54(14,7)$ & 1,18 & $0,85-1,62$ & \\
\hline
\end{tabular}

(IC95\%: 1,08-6,22). Na análise da variável escolaridade, observou-se que todas as categorias apresentaram valores estatisticamente significativos (Tabela 1).

A análise bivariada não mostrou diferenças estatisticamente significativas entre o uso de serviços de saúde e as variáveis: idade, cor da pele, estado civil, fumo, consumo de álcool, atividade física e em relação às morbidades investigadas neste estudo.
Deve ser mencionado que as mulheres que apresentavam hipertensão arterial apresentavam prevalências de não uso dos serviços de saúde aproximadamente $50 \%$ maior do que as sem a doença, contudo o limite inferior do intervalo de confiança foi o valor unitário, e a significância estatística foi maior do que 0,05.

As razões de prevalência e os respectivos intervalos de confiança foram estabelecidos levando-se em consideração o efeito de delineamento que foi de 0,98 . 
Algumas características das mulheres que não utilizam serviços de saúde segundo hábitos de vida e variáveis de morbidade. São Leopoldo, Rio Grande do Sul, Brasil, 2003.

\begin{tabular}{|c|c|c|c|c|c|}
\hline Variável & n (\%) & $\begin{array}{l}\text { Prevalência de } \\
\text { não uso (\%) }\end{array}$ & $\begin{array}{c}\text { Razão de } \\
\text { prevalência }\end{array}$ & IC95\% & Valor de $p$ \\
\hline Tabagismo & & & & & 0,78 \\
\hline Não & $603(59,0)$ & $85(14,1)$ & 1,00 & - & \\
\hline Ex-fumante & $188(18,4)$ & $18(9,6)$ & 0,68 & $0,42-1,10$ & \\
\hline Fumante & $231(22,6)$ & $33(14,3)$ & 1,01 & $0,70-1,47$ & \\
\hline Consumo de álcool (g etanol/dia) & & & & & 1,0 \\
\hline$<30$ & $962(94,1)$ & $128(13,3)$ & 1,00 & - & \\
\hline$\geq 30$ & $60(5,9)$ & $8(13,3)$ & 1,00 & $0,52-1,95$ & \\
\hline Atividade física & & & & & 0,26 \\
\hline Sim & $322(31,5)$ & $49(15,2)$ & 1,00 & - & \\
\hline Não & $700(68,5)$ & $874(12,4)$ & 0,82 & $0,59-1,13$ & \\
\hline Hipertensão arterial & & & & & 0,08 \\
\hline Não & $889(87,3)$ & $111(12,5)$ & 1,00 & - & \\
\hline Sim & $129(12,7)$ & $24(18,6)$ & 1,49 & $1,00-2,22$ & \\
\hline Diabetes mellitus & & & & & 0,78 \\
\hline Não & $985(96,4)$ & $130(13,2)$ & 1,00 & - & \\
\hline Sim & $37(3,6)$ & $6(16,2)$ & 1,23 & $0,58-2,60$ & \\
\hline Excesso de peso & & & & & 0,26 \\
\hline Não & $504(50,8)$ & $61(12,1)$ & 1,00 & - & \\
\hline Sim & $488(49,2)$ & $72(14,8)$ & 1,22 & $0,89-1,67$ & \\
\hline Distúrbios psiquiátricos menores & & & & & 0,65 \\
\hline Não & $586(57,4)$ & $81(13,8)$ & 1,00 & - & \\
\hline Sim & $435(42,6)$ & $55(12,6)$ & 0,91 & $0,66-1,26$ & \\
\hline
\end{tabular}

Na análise multivariável, através da regressão de Poisson, verificou-se que as mulheres pertencentes às classes $\mathrm{C}, \mathrm{D}$ e $\mathrm{E}$ tinham mais do que o dobro de probabilidades de não utilizarem serviços de saúde comparadas às da classe A. A prevalência de não uso dos serviços ambulatoriais de saúde pelas mulheres com hipertensão arterial sistêmica foi $41 \%$ maior do que as inseridas na categoria de base, contudo o limite inferior do intervalo de confiança foi menor do que o valor unitário. No segundo modelo, constatou-se que as mulheres com escolaridade mais baixa apresentavam maior probabilidade de não consultarem. Nesse modelo, os intervalos de confiança e o teste estatístico mostraram que as mulheres com hipertensão arterial ajustada para escolaridade apresentavam menor probabilidade de não consultarem. No terceiro modelo, apesar de o teste estatístico apresentar associação entre renda familiar ajustada para hipertensão arterial, os intervalos de confiança não confirmaram a relação. As mulheres com hipertensão arterial apresentaram uma probabilidade $44 \%$ maior de não consultarem com médico (Tabela 4).

\section{Discussão}

Os sistemas tradicionais de informação em saúde podem propiciar dados sobre as ações de saúde a partir dos atendimentos realizados. Porém, sofrem de limitações como, por exemplo, o desconhecimento das características daqueles que não procuram os serviços de saúde. Portanto, os estudos de prevalência com base populacional apresentam a vantagem de, quando representativos, possibilitarem estimativas do comportamento epidemiológico para toda a comunidade em que foram realizados.

Este estudo transversal foi representativo da população de São Leopoldo, pois os intervalos de confiança dos percentuais das distribuições por idade coincidiram com a distribuição da população do município verificada no recenseamento de 2000 , conferindo validade externa aos resultados.

As características definidoras da qualidade de serviços de saúde do ponto de vista coletivo são: o acesso facilitado, a distribuição eqüitativa da atenção e a efetividade dos cuidados a um 
Análise de regressão de Poisson dos fatores associados à utilização de serviços de saúde. São Leopoldo, Rio Grande do Sul Brasil, 2003

\begin{tabular}{|c|c|c|c|}
\hline Variável & Razão de prevalência & IC95\% & Valor de $p$ \\
\hline \multicolumn{4}{|l|}{ Modelo 1 * } \\
\hline Classe econômica ** & & & $<0,01$ \\
\hline A & 1,00 & - & \\
\hline B & 1,17 & $0,48-2,90$ & \\
\hline $\mathrm{C}$ & 2,31 & $1,02-5,22$ & \\
\hline$D+E$ & 2,40 & $1,04-5,54$ & \\
\hline Hipertensão arterial & & & 0,06 \\
\hline Não & 1,00 & - & \\
\hline Sim & 1,41 & $0,99-2,02$ & \\
\hline \multicolumn{4}{|l|}{ Modelo $2 * \star \star$} \\
\hline Escolaridade (anos) & & & 0,006 \\
\hline 12 ou mais & 1,00 & - & \\
\hline $8-11$ & 1,84 & $1,06-3,20$ & \\
\hline $5-7$ & 1,93 & $1,25-2,98$ & \\
\hline $0-4$ & 2,04 & $1,35-3,08$ & \\
\hline Hipertensão arterial & & & $<0,05$ \\
\hline Não & 1,00 & - & \\
\hline Sim & 1,27 & $1,35-3,08$ & \\
\hline \multicolumn{4}{|l|}{ Modelo 3 \# } \\
\hline Renda familiar (salários mínimos) & & & 0,003 \\
\hline 6,01 ou mais & 1,00 & - & \\
\hline $3,01-6$ & 0,81 & $0,20-3,26$ & \\
\hline $1,01-3$ & 1,86 & $0,65-5,34$ & \\
\hline $0-1$ & 2,49 & $0,88-7,00$ & \\
\hline Hipertensão arterial & & & $<0,05$ \\
\hline Não & 1,00 & - & \\
\hline Sim & 1,44 & $1,00-2,06$ & \\
\hline
\end{tabular}

* Classe econômica e hipertensão arterial ajustadas entre si;

** Segundo a Associação Nacional de Empresas de Pesquisa (ANEP) 13;

*** Escolaridade e hipertensão arterial ajustadas entre si;

\# Renda familiar e hipertensão arterial ajustadas entre si.

custo que seja suportável para a sociedade 20 . Considerou-se o uso de serviços ambulatoriais de saúde como uma condição que evidenciava, pelo menos, o acesso à rede básica em São Leopoldo. Assim, tentou-se revelar as características das mulheres que não utilizavam os serviços para identificar grupos populacionais excluídos e contribuir para o planejamento e a programação de atividades em saúde a partir da realidade encontrada.

A partir dos resultados encontrados, 13,3\% das mulheres não utilizaram os serviços de saúde. As comparações dos nossos resultados foram prejudicadas, pois outros estudos apresentaram critérios de utilização e populações diferentes. Por exemplo, em estudo transversal realizado na cidade de Pelotas, Rio Grande do Sul, no ano de
1992, 30\% dos indivíduos de 20 a 69 anos, de ambos os sexos, não utilizaram os serviços de saúde no período de um ano ${ }^{8}$. Outro estudo transversal de base populacional, realizado na cidade de Rio Grande, Rio Grande do Sul, incluindo 1.260 sujeitos com 15 anos ou mais, de ambos os sexos, mostrou que $66 \%$ utilizaram serviços de saúde no último ano 21. A Pesquisa Nacional por Amostra de Domicílios (PNAD), realizada em 1998, investigou o uso de serviço de saúde nos últimos 15 dias que antecederam a entrevista, os resultados mostraram que as mulheres de 25 a 49 anos apresentaram 17,1\% de utilização, e as de 50 a 64 anos, $22,2 \% 4$.

Tentou-se estabelecer a associação com alguns fatores sabidamente relacionados com o uso de serviços de saúde. Estudos têm mostra- 
do que nível sócio-econômico, sexo, idade, cor da pele, presença de companheiro, condições de saúde, existência de procura regular de serviços de saúde são fatores associados à utilização de serviços de saúde 5,22,23,24,25,26.

Contudo, no presente estudo, essas associações não foram estabelecidas. Exceto em relação às variáveis sócio-econômicas e hipertensão arterial sistêmica. Sabe-se que o acesso universal ao atendimento à saúde de elevada qualidade atenua as diferenças entre pobres e ricos 27 . Existem evidências revelando que a atenção básica ajuda a prevenir morbimortalidade e que é responsável pela distribuição mais equânime de saúde entre populações 28 . O conceito de eqüidade pressupõe tratar de forma prioritária as populações mais vulneráveis socialmente ou que apresentam reconhecidos riscos à saúde 29 . No presente estudo, constatou-se que as mulheres pertencentes às classes $\mathrm{C}, \mathrm{D}$ e E, com baixa renda per capita e escolaridade, ou seja, pessoas com maiores necessidades e mais vulneráveis, apresentaram menor utilização dos serviços de saúde, sugerindo condições desfavoráveis no sistema de saúde local, apontando para a presença de falta de universalidade e iniqüidade no acesso.

Sabe-se que o manejo das doenças crônicas não transmissíveis exige controle mais intensivo de pacientes aumentando inclusive seus contatos com os serviços de saúde. Estudos sobre uso de serviços de saúde mostraram associação positiva com presença ou referência de hipertensão arterial 30,31. No presente estudo, mesmo após controle para classe econômica, constatou-se que os pacientes com hipertensão arterial sistêmica utilizaram menos os serviços de saúde, o que pode revelar falta de organização dos serviços ou problemas na aderência dos pacientes.

Espera-se que os dados encontrados sirvam de parâmetros de comparação para o sistema de saúde do Município de São Leopoldo que apresenta, no momento, gestão plena e está ampliando o Programa Saúde da Família.

\section{Resumo}

O objetivo do estudo foi verificar a utilização dos serviços ambulatoriais de saúde e fatores associados pelas mulheres de 20 a 60 anos, residentes na zona urbana de São Leopoldo, Rio Grande do Sul, Brasil. Foi um estudo transversal de base populacional, incluindo 1.026 mulheres, no ano 2003. A associação entre as variáveis e o desfecho foi avaliada por meio das razões de prevalência e dos intervalos de $95 \%$ de confiança (IC95\%). A regressão de Poisson foi utilizada segundo modelo hierarquizado. Entre 1.022 mulheres, 136 (13,3\%; IC95\%: 11,2-15,4) não utilizaram os serviços ambulatoriais de saúde nos últimos 12 meses que precederam a entrevista. Demonstrou-se que as mulheres inseridas nas classes econômicas $C, D$ e E, com menos anos de estudo e com renda mais baixa apresentaram menor utilização dos serviços de saúde. Assim, as mulheres com maiores necessidades e mais vulneráveis apresentaram menor utilização dos serviços de saúde, sugerindo condições desfavoráveis no sistema de saúde local, apontando para a presença de falta de universalidade e iniqüidade no acesso.

Serviços Ambulatoriais de Saúde; Acesso aos Serviços de Saúde; Mulheres

\section{Colaboradores}

J. S. Dias-da-Costa e M. T. A. Olinto elaboraram o projeto de pesquisa, participaram da análise e redação do artigo. A. D. Presser, A. F. Zanolla, D. G. Ferreira, G. Perozzo, I. B. A. Freitas, L. T. Portolan, M. P. Pattussi e R. T. Tavares participaram da análise e redação do artigo.

\section{Agradecimentos}

À Fundação de Amparo à Pesquisa do Estado do Rio Grande do Sul (FAPERGS) via Proad2 (no. 02/0645,9) e Edital Universal 6 do Conselho Nacional de Desenvolvimento CientíficoeTecnológico (CNPq; $\left.\mathrm{n}^{\circ} .473478 / 200-0\right)$ pelo financiamento, e ao apoio da Secretaria Municipal de Saúde de São Leopoldo. 


\section{Referências}

1. Instituto Brasileiro de Geografia e Estatística. Estatísticas da saúde: assistência médico-sanitária. Rio de Janeiro: Instituto Brasileiro de Geografia e Estatística; 2006.

2. Pestana M, Mendes EV. Pacto de gestão: da municipalização autárquica à regionalização cooperativa. Belo Horizonte: Secretaria de Estado de Saúde de Minas Gerais; 2004.

3. Travassos C, Martins M. Uma revisão sobre os conceitos de acesso e utilização de serviços de saúde. Cad Saúde Pública 2004; 20 Suppl 2:S190-8.

4. Travassos C, Viacava F, Pinheiro R, Brito A. Utilização dos serviços de saúde no Brasil: gênero, características familiares e condição social. Rev Panam Salud Pública 2002; 11:365-73.

5. Sawyer DO, Leite IC, Alexandrino R. Perfis de utilização de serviços de saúde no Brasil. Ciênc Saúde Coletiva 2002; 7:757-76.

6. Goldbaum M, Gianini JR, Novaes HMD, César CLG Utilização de serviços de saúde em áreas cobertas pelo programa saúde da família (QUALIS) no Município de São Paulo. Rev Saúde Pública 2005; 39:90-9.

7. Mendoza-Sassi R, Béria JU. Utilización de los servicios de salud: una revisión sistemática sobre los factores relacionados. Cad Saúde Pública 2001; 17:819-32.

8. Dias-da-Costa JS, Facchini LA. Utilização de serviços ambulatoriais em Pelotas: onde a população consulta e com que freqüência. Rev Saúde Pública 1997; 31:360-9.

9. Lessa I, Mendonça GA, Teixeira MTB. Doenças crônicas não transmissíveis no Brasil: dos fatores de risco ao impacto social. Bol Oficina Sanit Panam 1996; 120:389-413.

10. Sá DA, Gouveia AC, Medeiros KR, Lyra TM. Avaliação da universalidade do acesso à saúde através dos sistemas de informações assistenciais do SUS: Brasil, 1995 e 1998. Rev Bras Saúde Matern Infant 2001; 1:45-52.

11. Lobato LVC. Reforma sanitária e reorganização do sistema de serviços de saúde: efeitos sobre a cobertura e a utilização de serviços [Tese de Doutorado]. Rio de Janeiro: Escola Nacional de Saúde Pública, Fundação Oswaldo Cruz; 2000.

12. Barros FC, Victora CG, Vaughan JP. Epidemiologia da saúde infantil: um manual para diagnósticos comunitários. São Paulo: Editora Hucitec/Brasília: Fundo das Nações Unidas para a Infância; 1990

13. Associação Nacional de Empresas de Pesquisa. Critério de classificação econômica Brasil. http:// www.anep.org.br/ (acessado em 23/Mai/2005).

14. Secretaria de Políticas de Saúde, Ministério da Saúde. Programa Nacional de Promoção da Atividade Física "Agita Brasil": atividade física e sua contribuição para a qualidade de vida. Rev Saúde Pública 2002; 36:254-6.

15. Moreira LB, Fuchs FD, Moraes RS, Bredemeier M, Cardozo S, Fuchs SC, et al. Alcoholic beverage consumption and associated factors in Porto Alegre, a southern Brazilian city: a population-based survey. J Stud Alcohol 1996; 57:253-9.
16. Chobanian AV, Bakris GL, Black HR, Cushman WC, Green LA, Izzo Jr. JL, et al. The Seventh Report of the Joint National Committee on Prevention, Detection, Evaluation, and Treatment of High Blood Pressure: the JNC 7 report. JAMA 2003; 289: 2560-72.

17. Mari JJ, Williams P. A validity study of a psychiatric screening questionnaire (SRQ-20) in primary care in the city of São Paulo. Br J Psychiatry 1986; 148:23-6.

18. Barros AJD, Hirakata VN. Alternatives for logistic regression in cross-sectional studies: an empirical comparison of models that directly estimate the prevalence ratio. BMC Med Res Methodol 2003; $3: 21$.

19. Victora CG, Huttly SR, Fuchs SC, Olinto MTA. The role of conceptual frameworks in epidemiological analysis: a hierarchical approach. Int J Epidemiol 1997; 26:224-47.

20. Campbell SM, Roland MO, Buetow SA. Defining quality of care. Soc Sci Med 2000; 51:1611-25.

21. Mendonza-Sassi R, Béria JU, Barros AJD. Outpatient health service utilization and associated factors: a population-based study. Rev Saúde Pública 2003; 37:372-8.

22. Culler SD, Parchman ML, Przbylsky M. Factors related to potentially preventable hospitalizations among elderly. Med Care 1998; 36:804-17.

23. Billings J, Zeitel L, Lukomnik J, Carey TS, Blank AE, Newman L. Impact of socioeconomic status on hospital use in New York City. Health Aff (Millwood) 1993; 12:162-73.

24. Castro MSM, Travassos C, Carvalho MS. Fatores associados às internações hospitalares no Brasil. Ciênc Saúde Coletiva 2002; 7:795-811.

25. Pinheiro RS, Viacava F, Travassos V, Brito AS. Gênero, morbidade, acesso e utilização de serviços de saúde no Brasil. Ciênc Saúde Coletiva 2002; 7:687707.

26. Castro MSM, Travassos C, Carvalho MS. Efeito da oferta de serviços de saúde no uso de internações hospitalares no Brasil. Rev Saúde Pública 2005; 39:277-84.

27. Marmot M. Health in an unequal world. Lancet 2006; 368:2081-94.

28. Starfield B, Shi L, Macinko J. Contribution of primary care to health systems and health. Milbank Q 2005; 83:457-502.

29. Wagstaff A. Poverty and health sector inequalities. Bull World Health Organ 2002; 80:97-105.

30. Barros MBA, César LGC, Carandina L, Torre GD. Desigualdades sociais na prevalência de doenças crônicas no Brasil, PNAD-2003. Ciênc Saúde Coletiva 2006; 11:911-26.

31. Lima-Costa MF, Barreto SM, Giatti L. Condições de saúde, capacidade funcional, uso de serviços de saúde e gastos com medicamentos da população idosa brasileira: um estudo descritivo baseado na Pesquisa Nacional por Amostras de Domicílios. Cad Saúde Pública 2003; 19:735-43.

Recebido em 14/Ago/2007

Versão final reapresentada em 22/Abr/2008

Aprovado em 07/Mai/2008 